\title{
Different supramolecular interactions mediated by bromine atoms in the crystal structures of three anisole derivates
}

\author{
Robert Nestler, ${ }^{a}$ Anke Schwarzer ${ }^{\mathrm{a}}$ and Tobias Gruber ${ }^{\mathrm{b} *}$

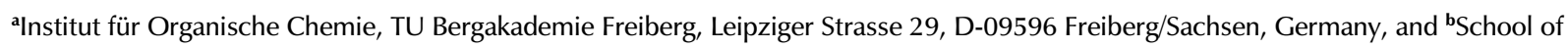 \\ Pharmacy, University of Lincoln, Joseph Banks Laboratories, Green Lane, Lincoln, LN6 7DL, United Kingdom \\ Correspondence email: tgruber@lincoln.ac.uk
}

\begin{abstract}
Three anisole building blocks featuring bishydroxymethyl or bisbromomethyl pendants have analyzed with regard to their molecular structure and packing behaviour. A typical supramolecular pattern we found were $\mathrm{C}-\mathrm{H} \cdots \pi$ interactions responsible for the generation of molecular stacks. $\pi \cdots \pi$ Interactions were only observed in the absence of bromine indicating a striking influence on the distances between adjacent aromatic moieties. When comparing the bishydroxymethyl compound with the respective bisbromomethyl compound we found that the strong $\mathrm{O}-\mathrm{H} \cdots \mathrm{O}$ in the zigzag arrangement in the first is replaced by $\mathrm{C}-\mathrm{H} \cdots \mathrm{Br}$ interactions in the second without changing the general packing.
\end{abstract}

\section{Introduction}

Phenols and their ethers are important building blocks for the a large variety of target compounds. Of crucial importance in this respect is their activation in order to allow the desired reactions. One way of preparing suited phenol monomers is the introduction of hydroxymethyl residues, which can be done by applying formaldehyde under basic conditions. For further use, the alcoholic $\mathrm{OH}$ groups can be transformed into bromomethyl groups (Moran et al., 1952).

During our studies on phenolic macrocycles we prepared the three title compounds, (I)-(III) and determined their molecular structures. All three are important educts for a wide range of molecules and materials. Title compound (I) has been used in the synthesis of selenacalixarenes (Thomas et al., 2012), title compounds (II) and (III) in the preparation of rotaxanes (Hirose et al., 2007a; Hirose et al., 2007b), supramolecular ligands (Li et al., 1988; Xu et al., 2012;

Sookcharoenpinyo et al., 2012), cyclophanes (Yamato et al., 2002; Yamato et al., 2006; Shimizu et al., 2009), macrocycles (Akine et al., 2000; Sharghi et al., 2001; Akine et al., 2005), crown ethers (Hirose et al., 2003) and pyrene derivatives (Tashiro et al., 1989).

\section{Experimental}

\subsection{Synthesis and crystallization}

Ethyl 3,5-bis(hydroxymethyl)-4-methoxy-benzoate, (I), has been synthesized from ethylparaben (Haba et al., 2005; Thomas et al., 2012). Title compounds (II), 4-bromo-2,6-bis(hydroxymethyl)anisole, and (III), 4-bromo-2,6-bis(bromomethyl)anisole, have been prepared from 4-bromophenol according to literature procedures (Leroy et al., 1988; Tashiro et al., 1989; González-Bulnes et al., 2013). Crystals suitable for X-ray diffraction were obtained by crystallization of the title compounds from the respective solvents at room temperature [(I): ethanol, m.p. $67^{\circ} \mathrm{C}$; (II): water, m.p. $127^{\circ} \mathrm{C}$; (III): ethanol, m.p. $\left.85^{\circ} \mathrm{C}\right]$.

\subsection{Refinement}

Data collection was performed at different temperatures [(I): $203 \mathrm{~K}$, (II): $173 \mathrm{~K}$, (III): $153 \mathrm{~K}$ ] due to instability of the single crystals and to avoid unintended ice formation during the measurements. Crystal data, data collection and structure refinement details are summarized in Table 1. 


\section{Results and discussion}

The title compounds (I-III) were found to crystallise with one molecule in the asymmetric part of the unit cell. While (I) crystallised in the triclinic space group $P-1$, (II) was detected in the noncentrosymmetric orthorhombic space group Pna $2_{1}$ and (III) in the monoclinic group $C 2 / c$. For all crystal structures no solvent accessible area was found (Spek, 2009). The packing indeces were calculated to 71.1 (I), 70.5 (II), and 70.2 (III) using Platon software (Spek, 2009). As expected the value slightly decreases with a higher number of bromine atoms.

First, the carbonyl group in the structure of (I) is more or less coplanar with the aromatic unit [dihedral angle of $\left.6.69(15)^{\circ}\right]$. The methoxy group is not coplanr and shows torsion angles of $83.7(2)^{\circ}$ and $-101.3(2)^{\circ}$ (see Table 2 for selected torsion angles). In contrast, both $\mathrm{CH}_{2} \mathrm{OH}$ substituents differ in their position relative to the benzene core. While the hydroxymethylene unit of $\mathrm{O} 1$ is almost coplanar $\left[4.6(3)^{\circ}\right.$ and $\left.-173.24(17)^{\circ}\right]$, the group of $\mathrm{O} 3$ gives a small torsion of about $-22.3(3)^{\circ}$ and $155.76^{\circ}$. This is similar to the orientation of the $\mathrm{CH}_{2} \mathrm{OH}$ groups in the structure of the free acid with torsion angles of $1.2^{\circ} / 179.1^{\circ}$, and $-41.6^{\circ} / 138.4^{\circ}$ for the second $\mathrm{CH}_{2} \mathrm{OH}$ group, respectively (Kohmoto et al., 2009). An inversed situation for one $\mathrm{CH}_{2} \mathrm{OH}$ group is observed in the crystal structure of methyl 3,5-bis(hydroxymethyl)benzoate (Katzsch et al., 2012). In here the torsion angles are $-174.7^{\circ} / 5.8^{\circ}$, and $-47.9^{\circ} / 131.6^{\circ}$ for the second $\mathrm{CH}_{2} \mathrm{OH}$ group. In the crystal structure of bromoanisol (II), the hydroxymethylene and methoxy substitutents are orientated like discribed for (I) with slight differences. The corresponding torsion angles are given in Table 3. The molecular geometry of compound (III) is described by the same orientation of the methoxy group as observed for (I) and (II). In constrast, the bromomethyl moieties point to different directions, ("trans" form). The first methylene unit at $\mathrm{Br} 1$ is orientated as $\mathrm{CH}_{2} \mathrm{OH}$ of $\mathrm{O} 1$ in (I) but with a greater torsion (Table 4). The second methylene moiety at Br3 shows an inversed orientation with a greater torsion compared to $\mathrm{O} 2$ in (I). A similar situation is found in related compounds such as 1-bromo-3,5-bis(bromomethyl)benzene with torsion angles of $77.4^{\circ} / 103.5^{\circ}$ and $106^{\circ} / 74.8^{\circ}$ (Jones et al., 2012) or 1,3-bis(bromomethyl)benzene with an inversed orientation but torsion angles of $-83.3^{\circ} / 97.5^{\circ}$ and $-82.9^{\circ} / 96.2^{\circ}$ (Jones \& Kus, 2007).

All three compounds (I)-(III) bear a methoxy group being able to form weak intermolecular hydrogen bonds. While (I) and (II) have two additional $\mathrm{CH}_{2} \mathrm{OH}$ group being known to give strong hydrogen bond networks, (III) does not. Furthermore, (I) possesses COOEt group which could participate in $\mathrm{C}-\mathrm{H}^{\cdots} \mathrm{O}$ or $\mathrm{O}-\mathrm{H} \cdots \mathrm{O}$ interactions. Indeed, the carbonyl oxygen atom $\mathrm{O} 4$ interacts in an $\mathrm{O}-\mathrm{H} \cdots \mathrm{O}$ hydrogen bond [O3- $\mathrm{H} 3 \mathrm{O} \cdots \mathrm{O} 4: 1.83$ (3) $\AA$, Table 3] connecting two adjacent stacking like chains. These stacks arises as follows. Two molecules of (I) are connected via $\mathrm{O}-\mathrm{H} \cdots \mathrm{O}$ hydrogen bonds between the two hydroxyl functionalities [O1- $\mathrm{H} 1 \mathrm{O} \cdots \mathrm{O} 3: 1.87$ (3) $\AA$, Table 3) to form discrete dimers, which are stabilized by $\pi^{\cdots} \pi$ stacking interactions $[\mathrm{d}=3.4815(16) \AA]$ (Fig. 1b). These dimers are connected via $\mathrm{C}-$ $\mathrm{H} \cdots \pi$ interaction of $2.92 \AA$ resulting in a chain-like stacking along the crystallographic $a$-axis. A weak $\mathrm{C}-\mathrm{H} \cdots \mathrm{O}$ contact of $2.65 \AA$ (Table 3) further stabilizes the molecular packing.

By way of contrast, as a consequence of the inversed orientation of the $\mathrm{CH}_{2} \mathrm{OH}$ group in methyl 3,5-bis(hydroxymethyl)benzoate (Katzsch et al., 2012), the hydrogen bonding pattern changes from discret dimers to a continous hydrogen bonding. The crystal structure of the free acid of (I) is dominated by the dimers resulting from the $\mathrm{COOH}$ group.

The molecule of the structure of (II) has two $\mathrm{CH}_{2} \mathrm{OH}$ group being able to interact in hydrogen bonds as observed in (I). In contrast to (I), no discrete dimers are formed but both hydroxyl groups are involved in linear $\mathrm{O}-\mathrm{H} \cdots \mathrm{O}$ hydrogen bonds. While $\mathrm{O} 1-\mathrm{H} 1 \cdots \mathrm{O} 3$ leads to a chain along the crystallographic $a$-axis, the combination with $\mathrm{O} 3-\mathrm{H} 3 \cdots \mathrm{O} 1$ results in a layerlike arrangement of stacked molecules within the $a c$-plane. $\mathrm{C}-\mathrm{H} \cdots \pi$ interactions of $2.82 \AA$ support the stacking along the $c$-axis. Adjacent layers are connected via $\mathrm{Br} \cdots \mathrm{Br}$ contacts and weak $\mathrm{C}-\mathrm{H} \cdots \mathrm{O}$ contacts $(>2.8 \AA)$. Very weak $\mathrm{C}-\mathrm{H} \cdots \mathrm{Br}$ contacts are observed in addition to the $\mathrm{O} 1-\mathrm{H} 1 \cdots \mathrm{O} 3$ hydrogen bond supporting the formation of 
chains. In contrast to (I), $\pi^{\cdots} \pi$ stacking interactions with distances between adjacent centres of the aromatic units of $>4.4$ $\AA$ are not involved in the molecular packing of (II).

When comparing the structure of title compound (II) with the structure of the free phenol, i.e. 4-bromo-2,6-bis(hydroxymethyl)phenol (Crisp et al., 2000), the overall packing behaviour is similar. In both cases, the arene units form continuous stacks in which the anisol units of (II) have the same orientation in every layer, whereas in the phenol every second molecule is rotated by $180^{\circ}$. This feature allows the phenolic hydroxyl group to take part in a combination of linear and branched hydrogen bonds, though prevents $\mathrm{Br} \cdots \mathrm{Br}$ contacts as found in (II).

In the packing of (III) stacks along the crystallographic $b$-axis are observed. These stacks are generated by $\mathrm{C}-\mathrm{H} \cdots \pi$ $\left(2.88 \AA, 117^{\circ}\right)$ and $\mathrm{C}-\mathrm{H} \cdots \mathrm{Br}\left(2.94 \AA, 155.3^{\circ}\right)$ interactions, and are connected to an adjacent stack via an additional $\mathrm{C}-$ $\mathrm{H} \cdots \mathrm{Br}$ contact along the $c$-axis $\left(2.99 \AA, 154.2^{\circ}\right)$. This chain along the $c$-axis is supported by a very weak $\mathrm{Br} \cdots \mathrm{Br}$ contact $[\mathrm{C} 1-\mathrm{Br} 2 \cdots \mathrm{Br} 3-\mathrm{C} 9: 3.7187$ (8) $\AA, \mathrm{x},-\mathrm{y}+1, \mathrm{z}-1 / 2]$ resulting in a bifurcation at $\mathrm{Br} 3$. According to the respective angles of $96.02(13)^{\circ}$ and $169.89(13)^{\circ}$ it is a rather clear type II interaction (Mukherjee et al., 2014). As observed in the structure of (II), $\pi \cdots \pi$ stacking interactions are involved in the formation of the stacks. Additionally, the methoxy group in (III) leads to a reduced number and longer bromine $\cdots$ bromine contacts in comparison to 1-bromo-3,5-bis(bromomethyl)benzene. In both cases, the aromatic bound $\mathrm{Br}$ is involved. Worthnoting, (II) and (III) display rather similar packing motifs. The aromatic units are piled up connected via $\mathrm{C}-\mathrm{H} \cdots \pi$ interactions, while the interconnection is facilitated by either $\mathrm{O}-\mathrm{H} \cdots \mathrm{O}$ or $\mathrm{C}-\mathrm{H} \cdots \mathrm{Br}$ interactions (Fig. 5).

In summary, we have analyzed the molecular structure and the packing behaviour of three arene building blocks featuring bis(hydroxymethyl) or bis(bromomethyl) substituents. In all structures $\mathrm{C}-\mathrm{H} \cdots \pi$ interactions are responsible for the generation of stacks. Therefore a typical supramolecular pattern the $\mathrm{C}-\mathrm{H} \cdots \pi$ interaction can be considered, but in combination with strong $\mathrm{O}-\mathrm{H} \cdots \mathrm{O}$ hydrogen bonds between the hydroxyl groups. For the bis(bromomethyl) derivative (III) the $\mathrm{O}-\mathrm{H} \cdots \mathrm{O}$ interactions in the zigzag arrangement seems to be replaced by $\mathrm{C}-\mathrm{H} \cdots \mathrm{Br}$ interactions without changing the general packing mode. Finally, $\pi^{\cdots} \pi$ interactions were onyl observed in the absence of bromine (I) indicating that the larger bromine atom enlarges the distances between adjacent aromatic moieties.

\section{Table 1}

Experimental details

\section{(I)}

Crystal data

Chemical formul

$M_{\mathrm{r}}$

Crystal system, space group Triclinic, $P \overline{1}$

Temperature (K)

$a, b, c(\AA)$

$\alpha, \beta, \gamma\left(^{\circ}\right)$

$V\left(\AA^{3}\right)$

$Z$

Radiation type

$\mu\left(\mathrm{mm}^{-1}\right)$

Crystal size (mm)

Data collection

(14)

(9)

$588.15(14)$

2

Mo $K \alpha$

0.11
(II)

$\mathrm{C}_{9} \mathrm{H}_{11} \mathrm{BrO}_{3}$

247.09

Orthorhombic, Pna2 1

173

7.8601 (10), 8.2489 (11), 10.788313 .1596 (7), 16.2514 (9), 4.4735

81.538 (11), 72.352 (10), 61.930

$0.35 \times 0.32 \times 0.28$
(2)

$90,90,90$

$956.71(9)$

4

Mo $K \alpha$

4.27

$0.45 \times 0.27 \times 0.15$
(III)

$\mathrm{C}_{9} \mathrm{H}_{9} \mathrm{Br}_{3} \mathrm{O}$

372.89

Monoclinic, $C 2 / c$

153

33.315 (4), $4.3913(3), 15.9655$

(17)

90, $110.142(8), 90$

$2192.9(4)$

8

Mo $K \alpha$

11.00

$0.50 \times 0.11 \times 0.09$ 
Diffractometer

Absorption correction

$T_{\min }, T_{\max }$

No. of measured, independent and observed $[I>2 \sigma(I)]$

reflections

$R_{\text {int }}$

$(\sin \theta / \lambda)_{\max }\left(\AA^{-1}\right)$

Refinement

$R\left[F^{2}>2 \sigma\left(F^{2}\right)\right], w R\left(F^{2}\right), S$

No. of reflections

No. of parameters

No. of restraints

$\mathrm{H}$-atom treatment

$\Delta \rho_{\max }, \Delta \rho_{\min }\left(\mathrm{e} \AA^{-3}\right)$

Absolute structure

Absolute structure parameter-
STOE IPDS 2

diffractometer

$-$

$-$

4748, 2188, 1705

0.052

0.606

$0.050,0.140,1.12$

2188

164

0

$\mathrm{H}$ atoms treated by a mixture of independent and constrained refinement

$0.23,-0.26$

$-$
STOE IPDS 2

diffractometer

Integration

$X$-AREA (STOE)

$0.283,0.564$

4183, 1904, 1647

0.074

0.627

$0.065,0.190,1.10$

1904

127

1

$\mathrm{H}$ atoms treated by a mixture of independent and constrained refinement

$0.82,-1.22$

Classical Flack method preferred over Parsons because s.u. lower.

0.01 (5)
0.087

0.622

STOE IPDS 2T

diffractometer

Integration

$X$-AREA (STOE)

$0.008,0.076$

$5754,2197,1834$

$0.033,0.088,1.10$

2197

119

0

H-atom parameters constrained

$0.63,-0.60$

Computer programs: STOE X-AREA, STOE X-RED, SHELXL2012 (Sheldrick, 2012), SHELXL2014 (Sheldrick, 2015), XP (Sheldrick, 2008), WinGX (Farrugia, 2012), publCIF (Westrip, 2010), SHELXLE (Hübschle et al., 2011).

\section{Table 2}

Hydrogen-bond geometry $\left(\AA,^{\circ}\right)$ for (I)

\begin{tabular}{lllll}
\hline$D-\mathrm{H} \cdots A$ & $D-\mathrm{H}$ & $\mathrm{H} \cdots A$ & $D \cdots A$ & $D-\mathrm{H} \cdots A$ \\
\hline $\mathrm{O} 1-\mathrm{H} 1 O \cdots \mathrm{O}^{\mathrm{i}}$ & $0.92(3)$ & $1.87(3)$ & $2.788(2)$ & $176(3)$ \\
$\mathrm{O} 3-\mathrm{H} 3 O \cdots 4^{\mathrm{ii}}$ & $0.91(3)$ & $1.83(3)$ & $2.725(2)$ & $167(3)$ \\
$\mathrm{C} 9-\mathrm{H} 9 B \cdots \mathrm{O}^{\text {ii }}$ & 0.99 & 2.65 & $3.555(3)$ & 153 \\
\hline
\end{tabular}

Symmetry codes: (i) $-x+1,-y+1,-z+1$; (ii) $x-1, y+1, z$; (iii) $-x,-y+2,-z+1$.

\section{Table 3}

Hydrogen-bond geometry $\left(\AA,{ }^{\circ}\right)$ for (II)

\begin{tabular}{lllll}
\hline$D-\mathrm{H} \cdots A$ & $D-\mathrm{H}$ & $\mathrm{H} \cdots A$ & $D \cdots A$ & $D-\mathrm{H} \cdots A$ \\
\hline $\mathrm{C} 8-\mathrm{H} 8 A \cdots \mathrm{Br} 1^{\mathrm{i}}$ & 0.98 & 3.11 & $3.773(13)$ & 126 \\
$\mathrm{C} 8-\mathrm{H} 8 B \cdots \mathrm{Br} 1^{\mathrm{ii}}$ & 0.98 & 3.11 & $3.917(19)$ & 140 \\
$\mathrm{O} 1-\mathrm{H} 1 \cdots \mathrm{O} 33^{\mathrm{ii}}$ & $1.0(2)$ & $1.8(2)$ & $2.687(13)$ & $146(20)$ \\
$\mathrm{O} 3-\mathrm{H} 3 \cdots \mathrm{O} 1^{\mathrm{iii}}$ & $0.76(16)$ & $1.95(17)$ & $2.685(13)$ & $160(18)$ \\
\hline
\end{tabular}

Symmetry codes: (i) $-x+1 / 2, y+1 / 2, z+1 / 2$; (ii) $x-1 / 2,-y+3 / 2, z$; (iii) $x+1 / 2,-y+3 / 2, z-1$. 


\section{Table 4}

Hydrogen-bond geometry $\left(\AA,{ }^{\circ}\right)$ for (III)

\begin{tabular}{lllll}
\hline$D-\mathrm{H} \cdots A$ & $D-\mathrm{H}$ & $\mathrm{H} \cdots A$ & $D \cdots A$ & $D-\mathrm{H} \cdots A$ \\
\hline $\mathrm{C} 2-\mathrm{H} 2 \cdots \mathrm{Br}^{\mathrm{i}}$ & 0.95 & 2.99 & $3.863(5)$ & 154 \\
$\mathrm{C} 7-\mathrm{H} 7 A \cdots \mathrm{Br} 2^{\mathrm{ii}}$ & 0.99 & 2.94 & $3.866(5)$ & 155 \\
\hline
\end{tabular}

Symmetry codes: (i) $x,-y+1, z-1 / 2$; (ii) $x, y+1, z$.

\section{Acknowledgements}

Financial support from the 'Fonds der Chemischen Industrie' and the Institute of Organic Chemistry, University of Freiberg, is gratefully acknowledged.

\section{References}

Akine, S., Goto, K. \& Kawashima, T. (2000). Tetrahedron Lett. 41, 897-901.

Akine, S., Goto, K. \& Kawashima, T. (2005). Bull. Chem. Soc. Jpn. 78, 169-179.

Crisp, G. T., Turner, P. D. \& Tiekink, E. R. T. (2000). Z. Kristallogr. - New Cryst. Struct. 215, 443-444.

Farrugia, L. J. (2012). J. Appl. Cryst. 45, 849-854.

González-Bulnes, L., Ibáñez, I., Bedoya, L. M., Beltrán, M., Catalán, S., Alcamí, J., Fustero, S. \& Gallego, J. (2013). Angew. Chem. Int. Ed. 52, 13405-13409.

Haba, K., Popkov, M., Shamis, M., Lerner, R. A., Barbas, C. F. \& Shabat, D. (2005). Angew. Chem. 117, 726-730.

Hirose, K., Fujiwara, A., Matsunaga, K., Aoki, N. \& Tobe, Y. (2003). Tetrahedron: Asymmetry 14, 555-566.

Hirose, K., Ishibashi, K., Shiba, Y., Doi, Y. \& Tobe, Y. (2007b). Chem. Lett. 36, 810-811.

Hirose, K., Nishihara, K., Harada, N., Nakamura, Y., Masuda, D., Araki, M. \& Tobe, Y. (2007a). Org. Lett. 9, 2969-2972.

Hübschle, C. B., Sheldrick, G. M. \& Dittrich, B. (2011). J. Appl. Cryst. 44, 1281-1284.

Jones, P. G. \& Kus, P. (2007). Z. Naturforsch. B Chem. Sci. 62, 725-731.

Jones, P. G., Kus, P. \& Dix, I. (2012). Z. Naturforsch. B Chem. Sci. 67, 1273-1281.

Katzsch, F., Eissmann, D. \& Weber, E. (2012). Struct. Chem. 23, 245-255.

Kohmoto, S., Kuroda, Y., Someya, Y., Kishikawa, K., Masu, H., Yamaguchi, K. \& Azumaya, I. (2009). Cryst. Growth Des. 9, 3457-3462.

Leroy, J., Wakselman, C., Lacroix, P. \& Kahn, O. (1988). J. Fluorine Chem. 40, 23-32.

Li, Y., Wu, D., Zhu, J. \& Zhao, H. (1988). Youji Huaxue 8, 158-61.

Moran, W. J., Schreiber, E. C., Engel, E., Behn, D. C. \& Yamins, J. L. (1952). J. Am. Chem. Soc. 74, 127-129

Mukherjee, A., Tothadi, S. \& Desiraju, G. R. (2014). Acc. Chem. Res. 47, 2514-2524.

Sharghi, H., Ali Nasseri, M. \& Niknam, K. (2001). J. Org. Chem. 66, 7287-7293.

Sheldrick, G. M. (2008). Acta Cryst. A64, 112-122.

Sheldrick, G. M. (2015). Acta Cryst. C71, 3-8.

Shimizu, T., Maeda, T., Hida, K. \& Yamato, T. (2009). J. Chem. Res. 8, 515-519.

Sookcharoenpinyo, B., Klein, E., Ferrand, Y., Walker, D. B., Brotherhood, P. R., Ke, C., Crump, M. P. \& Davis, A. P. (2012). Angew. Chem., Int. Ed. 51, 4586-4590. 
Spek, A. L. (2009). Acta Cryst. D65, 148-155.

Tashiro, M., Mataka, S., Takezaki, Y., Takeshita, M., Arimura, T., Tsuge, A. \& Yamato, T. (1989). J. Org. Chem. 54, 451458.

Thomas, J., Dobrzanska, L., van Hecke, K., Sonawane, M. P., Robeyns, K., van Meervelt, L., Wozniak, K., Smet, M., Maes, W. \& Dehaen, W. (2012). Org. Biomol. Chem. 10, 6526-6536.

Westrip, S. P. (2010). J. Appl. Cryst. 43, 920-925.

Xu, L., Li, Y., Yu, Y., Liu, T., Cheng, S., Liu, H. \& Li, Y. (2012). Org. Biomol. Chem. 10, 4375-4380.

Yamato, T., Okabe, R., Miyamoto, S. \& Miyazaki, M. (2006). J. Chem. Res. 9, 593-595.

Yamato, T., Tsuchihashi, K., Nakamura, N., Hirahara, M. \& Tanaka, K. (2002). Can. J. Chem. 80, 510-516.

\section{Figure 1}

Perspective views of (I)-(III) showing 50\% probability displacement ellipsoids for the non-H atoms and the used numbering.

\section{Figure 2}

Least-squares overlays of a) (II) (black lines) and (I) (blue lines), b) (I) (black lines) and (III) (blue lines) and c) (II) (black lines) and (III) (blue lines) with the following r.m.s. deviations: a) $0.0198 \AA$, b) $0.0220 \AA$ and c) $0.0122 \AA$ for an overlay of the following atoms: $\mathrm{C} 1-\mathrm{C} 6$. All hydrogen atoms not bonded to oxygen are omitted for clarity. Relevant atoms are labelled with a) A (II) and B (I), b) A (I) and B (III) and c) A (II) and B (III).

\section{Figure 3}

Packing of I

\section{Figure 4}

Packing of II Packing of III

\section{Figure 5}

Schematic non-covalent interactions in the packing of (II) and (III). 


\section{supporting information}

\section{Different supramolecular interactions mediated by bromine atoms in the crystal structures of three anisole derivates}

\section{Robert Nestler, Anke Schwarzer and Tobias Gruber*}

\section{Computing details}

For all compounds, data collection: STOE $X$-AREA; cell refinement: STOE $X$-AREA; data reduction: STOE $X$-RED; program(s) used to solve structure: SHELXL2012 (Sheldrick, 2012); program(s) used to refine structure: SHELXL2014 (Sheldrick, 2015); molecular graphics: XP (Sheldrick, 2008); software used to prepare material for publication: WinGX (Farrugia, 2012), publCIF (Westrip, 2010), SHELXLE (Hübschle et al., 2011).

(I)

Crystal data

$\mathrm{C}_{12} \mathrm{H}_{16} \mathrm{O}_{5}$

$M_{r}=240.25$

Triclinic, $P \overline{1}$

$a=7.8601(10) \AA$

$b=8.2489(11) \AA$

$c=10.7883(14) \AA$

$\alpha=81.538(11)^{\circ}$

$\beta=72.352(10)^{\circ}$

$\gamma=61.930(9)^{\circ}$

$V=588.15(14) \AA^{3}$

$Z=2$

\section{Data collection}

STOE IPDS 2

diffractometer

Radiation source: sealed X-ray tube, 12 x $0.4 \mathrm{~mm}$ long-fine focus

Plane graphite monochromator Detector resolution: 6.67 pixels $\mathrm{mm}^{-1}$

rotation method scans

4748 measured reflections

Refinement

Refinement on $F^{2}$

Least-squares matrix: full

$R\left[F^{2}>2 \sigma\left(F^{2}\right)\right]=0.050$

$w R\left(F^{2}\right)=0.140$

$S=1.12$

2188 reflections

164 parameters

0 restraints
$F(000)=256$

$D_{\mathrm{x}}=1.357 \mathrm{Mg} \mathrm{m}^{-3}$

Melting point: $340.15 \mathrm{~K}$

Mo $K \alpha$ radiation, $\lambda=0.71073 \AA$

Cell parameters from 2183 reflections

$\theta=2.0-27.4^{\circ}$

$\mu=0.11 \mathrm{~mm}^{-1}$

$T=203 \mathrm{~K}$

Piece, colorless

$0.35 \times 0.32 \times 0.28 \mathrm{~mm}$

2188 independent reflections

1705 reflections with $I>2 \sigma(I)$

$R_{\text {int }}=0.052$

$\theta_{\max }=25.5^{\circ}, \theta_{\min }=2.0^{\circ}$

$h=-9 \rightarrow 9$

$k=-9 \rightarrow 9$

$l=-13 \rightarrow 13$

Hydrogen site location: mixed

$\mathrm{H}$ atoms treated by a mixture of independent and constrained refinement

$w=1 /\left[\sigma^{2}\left(F_{\mathrm{o}}^{2}\right)+(0.0651 P)^{2}+0.2206 P\right]$

where $P=\left(F_{\mathrm{o}}^{2}+2 F_{\mathrm{c}}{ }^{2}\right) / 3$

$(\Delta / \sigma)_{\max }<0.001$

$\Delta \rho_{\max }=0.23$ e $\AA^{-3}$

$\Delta \rho_{\min }=-0.26$ e $\AA^{-3}$ 


\section{Special details}

Geometry. All esds (except the esd in the dihedral angle between two l.s. planes) are estimated using the full covariance matrix. The cell esds are taken into account individually in the estimation of esds in distances, angles and torsion angles; correlations between esds in cell parameters are only used when they are defined by crystal symmetry. An approximate (isotropic) treatment of cell esds is used for estimating esds involving l.s. planes.

Refinement. $\mathrm{H}$ atoms were positioned geometrically and allowed to ride on their parent atoms, $\mathrm{C}-\mathrm{H}=0.95-0.98 \% \mathrm{~A}$ and $\mathrm{Uiso}(\mathrm{H})=$ 1.2-1.5 Ueq (parent atom). Hydrogen atoms bonded to $\mathrm{O}$ were located via electron density. Individual reflections were considered as outliers and therefore omitted.

Fractional atomic coordinates and isotropic or equivalent isotropic displacement parameters $\left(\AA^{2}\right)$ for (I)

\begin{tabular}{|c|c|c|c|c|}
\hline & $x$ & $y$ & $z$ & $U_{\text {iso }} * / U_{\text {eq }}$ \\
\hline $\mathrm{O} 1$ & $0.6177(2)$ & $0.0793(2)$ & $0.74711(15)$ & $0.0381(4)$ \\
\hline $\mathrm{H} 1 \mathrm{O}$ & $0.725(5)$ & $0.106(4)$ & $0.714(3)$ & $0.063(9)^{*}$ \\
\hline $\mathrm{O} 2$ & $0.1535(2)$ & $0.6048(2)$ & $0.72607(14)$ & $0.0332(4)$ \\
\hline $\mathrm{O} 3$ & $0.0670(2)$ & $0.8314(2)$ & $0.36301(15)$ & $0.0375(4)$ \\
\hline $\mathrm{H} 3 \mathrm{O}$ & $-0.053(5)$ & $0.906(4)$ & $0.345(3)$ & $0.068(9)^{*}$ \\
\hline $\mathrm{O} 4$ & $0.7377(2)$ & $0.0287(2)$ & $0.27189(14)$ & $0.0337(4)$ \\
\hline O5 & $0.5225(2)$ & $0.2543(2)$ & $0.17245(13)$ & $0.0333(4)$ \\
\hline $\mathrm{C} 1$ & $0.4636(3)$ & $0.2861(3)$ & $0.39511(18)$ & $0.0242(4)$ \\
\hline $\mathrm{C} 2$ & $0.5062(3)$ & $0.2150(3)$ & 0.51367 (19) & $0.0257(4)$ \\
\hline $\mathrm{H} 2$ & 0.6094 & 0.0941 & 0.5179 & $0.031^{*}$ \\
\hline $\mathrm{C} 3$ & $0.3986(3)$ & $0.3202(3)$ & $0.62487(19)$ & $0.0263(4)$ \\
\hline $\mathrm{C} 4$ & $0.2463(3)$ & $0.4967(3)$ & 0.61648 (19) & $0.0253(4)$ \\
\hline $\mathrm{C} 5$ & $0.1989(3)$ & $0.5687(3)$ & $0.49936(19)$ & $0.0255(4)$ \\
\hline C6 & $0.3094(3)$ & $0.4616(3)$ & $0.38892(19)$ & $0.0256(4)$ \\
\hline H6 & 0.2797 & 0.5082 & 0.3081 & $0.031 *$ \\
\hline $\mathrm{C} 7$ & $0.4465(3)$ & $0.2513(3)$ & $0.7539(2)$ & $0.0329(5)$ \\
\hline H7A & 0.4672 & 0.3432 & 0.7887 & $0.040 *$ \\
\hline H7B & 0.3298 & 0.2412 & 0.8159 & $0.040^{*}$ \\
\hline $\mathrm{C} 8$ & -0.0362 (4) & $0.6174(4)$ & $0.7980(3)$ & $0.0515(7)$ \\
\hline H8A & -0.0781 & 0.6774 & 0.8814 & $0.077^{*}$ \\
\hline H8B & -0.0270 & 0.4938 & 0.8139 & $0.077^{*}$ \\
\hline $\mathrm{H} 8 \mathrm{C}$ & -0.1345 & 0.6897 & 0.7486 & $0.077^{*}$ \\
\hline C9 & $0.0352(3)$ & $0.7610(3)$ & $0.4921(2)$ & $0.0324(5)$ \\
\hline H9A & -0.0962 & 0.7595 & 0.5193 & $0.039^{*}$ \\
\hline H9B & 0.0336 & 0.8418 & 0.5523 & $0.039^{*}$ \\
\hline $\mathrm{C} 10$ & $0.5892(3)$ & $0.1750(3)$ & 0.27619 (19) & $0.0270(4)$ \\
\hline $\mathrm{C} 11$ & $0.6403(4)$ & $0.1645(4)$ & $0.0483(2)$ & $0.0429(6)$ \\
\hline H11A & 0.5509 & 0.1828 & -0.0057 & $0.051 *$ \\
\hline H11B & 0.7165 & 0.0308 & 0.0621 & $0.051^{*}$ \\
\hline $\mathrm{C} 12$ & $0.7810(5)$ & $0.2422(5)$ & $-0.0196(3)$ & $0.0652(8)$ \\
\hline $\mathrm{H} 12 \mathrm{~A}$ & 0.8573 & 0.1841 & -0.1049 & $0.098^{*}$ \\
\hline $\mathrm{H} 12 \mathrm{~B}$ & 0.8732 & 0.2188 & 0.0324 & $0.098^{*}$ \\
\hline $\mathrm{H} 12 \mathrm{C}$ & 0.7052 & 0.3749 & -0.0312 & $0.098^{*}$ \\
\hline
\end{tabular}

Atomic displacement parameters $\left(\AA^{2}\right)$ for (I)

\begin{tabular}{lllllll}
\hline & $U^{11}$ & $U^{22}$ & $U^{33}$ & $U^{12}$ & $U^{13}$ & $U^{23}$ \\
\hline O1 & $0.0355(8)$ & $0.0385(9)$ & $0.0355(9)$ & $-0.0116(7)$ & $-0.0154(7)$ & $0.0076(7)$ \\
O2 & $0.0290(7)$ & $0.0382(8)$ & $0.0299(8)$ & $-0.0135(6)$ & $-0.0024(6)$ & $-0.0106(6)$
\end{tabular}




\begin{tabular}{lllllll}
\hline & & & & & \\
O3 & $0.0285(8)$ & $0.0354(8)$ & $0.0375(9)$ & $-0.0061(6)$ & $-0.0116(6)$ & $0.0070(7)$ \\
O4 & $0.0285(7)$ & $0.0314(8)$ & $0.0322(8)$ & $-0.0042(6)$ & $-0.0094(6)$ & $-0.0047(6)$ \\
O5 & $0.0330(8)$ & $0.0359(8)$ & $0.0234(7)$ & $-0.0083(6)$ & $-0.0095(6)$ & $-0.0007(6)$ \\
C1 & $0.0228(9)$ & $0.0250(10)$ & $0.0248(10)$ & $-0.0111(8)$ & $-0.0062(7)$ & $0.0002(8)$ \\
C2 & $0.0222(9)$ & $0.0250(10)$ & $0.0279(10)$ & $-0.0086(8)$ & $-0.0081(8)$ & $0.0011(8)$ \\
C3 & $0.0260(10)$ & $0.0297(10)$ & $0.0263(10)$ & $-0.0146(8)$ & $-0.0089(8)$ & $0.0019(8)$ \\
C4 & $0.0226(9)$ & $0.0289(10)$ & $0.0259(10)$ & $-0.0138(8)$ & $-0.0032(7)$ & $-0.0036(8)$ \\
C5 & $0.0236(9)$ & $0.0237(10)$ & $0.0300(10)$ & $-0.0104(8)$ & $-0.0086(8)$ & $0.0001(8)$ \\
C6 & $0.0261(9)$ & $0.0264(10)$ & $0.0262(10)$ & $-0.0123(8)$ & $-0.0103(8)$ & $0.0030(8)$ \\
C7 & $0.0321(11)$ & $0.0362(12)$ & $0.0266(11)$ & $-0.0115(9)$ & $-0.0097(8)$ & $0.0014(9)$ \\
C8 & $0.0346(12)$ & $0.0760(19)$ & $0.0401(14)$ & $-0.0267(12)$ & $0.0064(10)$ & $-0.0185(13)$ \\
C9 & $0.0298(10)$ & $0.0266(11)$ & $0.0337(11)$ & $-0.0083(8)$ & $-0.0071(8)$ & $0.0013(8)$ \\
C10 & $0.0244(9)$ & $0.0300(11)$ & $0.0264(10)$ & $-0.0121(9)$ & $-0.0069(8)$ & $0.0004(8)$ \\
C11 & $0.0477(13)$ & $0.0480(14)$ & $0.0236(11)$ & $-0.0126(11)$ & $-0.0083(9)$ & $-0.0084(10)$ \\
C12 & $0.0626(18)$ & $0.081(2)$ & $0.0404(15)$ & $-0.0330(16)$ & $0.0070(13)$ & $-0.0083(14)$ \\
& & & & & & \\
\hline
\end{tabular}

Geometric parameters $\left(\AA,{ }^{o}\right)$ for $(I)$

\begin{tabular}{|c|c|c|c|}
\hline $\mathrm{O} 1-\mathrm{C} 7$ & $1.415(3)$ & $\mathrm{C} 5-\mathrm{C} 6$ & $1.384(3)$ \\
\hline $\mathrm{O} 1-\mathrm{H} 1 \mathrm{O}$ & $0.92(3)$ & $\mathrm{C} 5-\mathrm{C} 9$ & $1.511(3)$ \\
\hline $\mathrm{O} 2-\mathrm{C} 4$ & $1.379(2)$ & $\mathrm{C} 6-\mathrm{H} 6$ & 0.9500 \\
\hline $\mathrm{O} 2-\mathrm{C} 8$ & $1.419(3)$ & $\mathrm{C} 7-\mathrm{H} 7 \mathrm{~A}$ & 0.9900 \\
\hline $\mathrm{O} 3-\mathrm{C} 9$ & $1.423(3)$ & $\mathrm{C} 7-\mathrm{H} 7 \mathrm{~B}$ & 0.9900 \\
\hline $\mathrm{O} 3-\mathrm{H} 3 \mathrm{O}$ & $0.91(3)$ & $\mathrm{C} 8-\mathrm{H} 8 \mathrm{~A}$ & 0.9800 \\
\hline $\mathrm{O} 4-\mathrm{C} 10$ & $1.216(2)$ & $\mathrm{C} 8-\mathrm{H} 8 \mathrm{~B}$ & 0.9800 \\
\hline $\mathrm{O} 5-\mathrm{C} 10$ & $1.332(2)$ & $\mathrm{C} 8-\mathrm{H} 8 \mathrm{C}$ & 0.9800 \\
\hline $\mathrm{O} 5-\mathrm{C} 11$ & $1.447(3)$ & C9- $\mathrm{H} 9 \mathrm{~A}$ & 0.9900 \\
\hline $\mathrm{C} 1-\mathrm{C} 6$ & $1.393(3)$ & C9- $\mathrm{H} 9 \mathrm{~B}$ & 0.9900 \\
\hline $\mathrm{C} 1-\mathrm{C} 2$ & $1.395(3)$ & $\mathrm{C} 11-\mathrm{C} 12$ & $1.486(4)$ \\
\hline $\mathrm{C} 1-\mathrm{C} 10$ & $1.479(3)$ & C11-H11A & 0.9900 \\
\hline $\mathrm{C} 2-\mathrm{C} 3$ & $1.381(3)$ & C11-H11B & 0.9900 \\
\hline $\mathrm{C} 2-\mathrm{H} 2$ & 0.9500 & $\mathrm{C} 12-\mathrm{H} 12 \mathrm{~A}$ & 0.9800 \\
\hline $\mathrm{C} 3-\mathrm{C} 4$ & $1.396(3)$ & $\mathrm{C} 12-\mathrm{H} 12 \mathrm{~B}$ & 0.9800 \\
\hline $\mathrm{C} 3-\mathrm{C} 7$ & $1.512(3)$ & $\mathrm{C} 12-\mathrm{H} 12 \mathrm{C}$ & 0.9800 \\
\hline $\mathrm{C} 4-\mathrm{C} 5$ & $1.395(3)$ & & \\
\hline $\mathrm{C} 7-\mathrm{O} 1-\mathrm{H} 1 \mathrm{O}$ & $105(2)$ & $\mathrm{O} 2-\mathrm{C} 8-\mathrm{H} 8 \mathrm{~A}$ & 109.5 \\
\hline $\mathrm{C} 4-\mathrm{O} 2-\mathrm{C} 8$ & $115.78(17)$ & $\mathrm{O} 2-\mathrm{C} 8-\mathrm{H} 8 \mathrm{~B}$ & 109.5 \\
\hline $\mathrm{C} 9-\mathrm{O} 3-\mathrm{H} 3 \mathrm{O}$ & $110(2)$ & $\mathrm{H} 8 \mathrm{~A}-\mathrm{C} 8-\mathrm{H} 8 \mathrm{~B}$ & 109.5 \\
\hline $\mathrm{C} 10-\mathrm{O} 5-\mathrm{C} 11$ & $117.40(16)$ & $\mathrm{O} 2-\mathrm{C} 8-\mathrm{H} 8 \mathrm{C}$ & 109.5 \\
\hline $\mathrm{C} 6-\mathrm{C} 1-\mathrm{C} 2$ & $120.16(17)$ & $\mathrm{H} 8 \mathrm{~A}-\mathrm{C} 8-\mathrm{H} 8 \mathrm{C}$ & 109.5 \\
\hline $\mathrm{C} 6-\mathrm{C} 1-\mathrm{C} 10$ & $120.83(17)$ & $\mathrm{H} 8 \mathrm{~B}-\mathrm{C} 8-\mathrm{H} 8 \mathrm{C}$ & 109.5 \\
\hline $\mathrm{C} 2-\mathrm{C} 1-\mathrm{C} 10$ & $118.96(17)$ & $\mathrm{O} 3-\mathrm{C} 9-\mathrm{C} 5$ & $110.39(17)$ \\
\hline $\mathrm{C} 3-\mathrm{C} 2-\mathrm{C} 1$ & $120.11(17)$ & $\mathrm{O} 3-\mathrm{C} 9-\mathrm{H} 9 \mathrm{~A}$ & 109.6 \\
\hline $\mathrm{C} 3-\mathrm{C} 2-\mathrm{H} 2$ & 119.9 & $\mathrm{C} 5-\mathrm{C} 9-\mathrm{H} 9 \mathrm{~A}$ & 109.6 \\
\hline $\mathrm{C} 1-\mathrm{C} 2-\mathrm{H} 2$ & 119.9 & $\mathrm{O} 3-\mathrm{C} 9-\mathrm{H} 9 \mathrm{~B}$ & 109.6 \\
\hline $\mathrm{C} 2-\mathrm{C} 3-\mathrm{C} 4$ & $118.91(18)$ & $\mathrm{C} 5-\mathrm{C} 9-\mathrm{H} 9 \mathrm{~B}$ & 109.6 \\
\hline $\mathrm{C} 2-\mathrm{C} 3-\mathrm{C} 7$ & $121.67(18)$ & $\mathrm{H} 9 \mathrm{~A}-\mathrm{C} 9-\mathrm{H} 9 \mathrm{~B}$ & 108.1 \\
\hline $\mathrm{C} 4-\mathrm{C} 3-\mathrm{C} 7$ & $119.38(17)$ & $\mathrm{O} 4-\mathrm{C} 10-\mathrm{O} 5$ & $123.23(18)$ \\
\hline $\mathrm{O} 2-\mathrm{C} 4-\mathrm{C} 5$ & $120.26(17)$ & $\mathrm{O} 4-\mathrm{C} 10-\mathrm{C} 1$ & $124.56(18)$ \\
\hline $\mathrm{O} 2-\mathrm{C} 4-\mathrm{C} 3$ & $117.72(18)$ & $\mathrm{O} 5-\mathrm{C} 10-\mathrm{C} 1$ & $112.21(16)$ \\
\hline
\end{tabular}




\begin{tabular}{|c|c|c|c|}
\hline $\mathrm{C} 5-\mathrm{C} 4-\mathrm{C} 3$ & $121.82(17)$ & $\mathrm{O} 5-\mathrm{C} 11-\mathrm{C} 12$ & $109.7(2)$ \\
\hline $\mathrm{C} 6-\mathrm{C} 5-\mathrm{C} 4$ & $118.35(17)$ & $\mathrm{O} 5-\mathrm{C} 11-\mathrm{H} 11 \mathrm{~A}$ & 109.7 \\
\hline $\mathrm{C} 6-\mathrm{C} 5-\mathrm{C} 9$ & $120.67(18)$ & $\mathrm{C} 12-\mathrm{C} 11-\mathrm{H} 11 \mathrm{~A}$ & 109.7 \\
\hline $\mathrm{C} 4-\mathrm{C} 5-\mathrm{C} 9$ & $120.96(18)$ & $\mathrm{O} 5-\mathrm{C} 11-\mathrm{H} 11 \mathrm{~B}$ & 109.7 \\
\hline $\mathrm{C} 5-\mathrm{C} 6-\mathrm{C} 1$ & $120.63(18)$ & $\mathrm{C} 12-\mathrm{C} 11-\mathrm{H} 11 \mathrm{~B}$ & 109.7 \\
\hline $\mathrm{C} 5-\mathrm{C} 6-\mathrm{H} 6$ & 119.7 & $\mathrm{H} 11 \mathrm{~A}-\mathrm{C} 11-\mathrm{H} 11 \mathrm{~B}$ & 108.2 \\
\hline $\mathrm{C} 1-\mathrm{C} 6-\mathrm{H} 6$ & 119.7 & $\mathrm{C} 11-\mathrm{C} 12-\mathrm{H} 12 \mathrm{~A}$ & 109.5 \\
\hline $\mathrm{O} 1-\mathrm{C} 7-\mathrm{C} 3$ & $114.12(17)$ & $\mathrm{C} 11-\mathrm{C} 12-\mathrm{H} 12 \mathrm{~B}$ & 109.5 \\
\hline $\mathrm{O} 1-\mathrm{C} 7-\mathrm{H} 7 \mathrm{~A}$ & 108.7 & $\mathrm{H} 12 \mathrm{~A}-\mathrm{C} 12-\mathrm{H} 12 \mathrm{~B}$ & 109.5 \\
\hline $\mathrm{C} 3-\mathrm{C} 7-\mathrm{H} 7 \mathrm{~A}$ & 108.7 & $\mathrm{C} 11-\mathrm{C} 12-\mathrm{H} 12 \mathrm{C}$ & 109.5 \\
\hline $\mathrm{O} 1-\mathrm{C} 7-\mathrm{H} 7 \mathrm{~B}$ & 108.7 & $\mathrm{H} 12 \mathrm{~A}-\mathrm{C} 12-\mathrm{H} 12 \mathrm{C}$ & 109.5 \\
\hline $\mathrm{C} 3-\mathrm{C} 7-\mathrm{H} 7 \mathrm{~B}$ & 108.7 & $\mathrm{H} 12 \mathrm{~B}-\mathrm{C} 12-\mathrm{H} 12 \mathrm{C}$ & 109.5 \\
\hline $\mathrm{H} 7 \mathrm{~A}-\mathrm{C} 7-\mathrm{H} 7 \mathrm{~B}$ & 107.6 & & \\
\hline $\mathrm{C} 6-\mathrm{C} 1-\mathrm{C} 2-\mathrm{C} 3$ & $-1.5(3)$ & $\mathrm{C} 9-\mathrm{C} 5-\mathrm{C} 6-\mathrm{C} 1$ & $178.35(18)$ \\
\hline $\mathrm{C} 10-\mathrm{C} 1-\mathrm{C} 2-\mathrm{C} 3$ & $176.25(17)$ & $\mathrm{C} 2-\mathrm{C} 1-\mathrm{C} 6-\mathrm{C} 5$ & $1.1(3)$ \\
\hline $\mathrm{C} 1-\mathrm{C} 2-\mathrm{C} 3-\mathrm{C} 4$ & $0.7(3)$ & $\mathrm{C} 10-\mathrm{C} 1-\mathrm{C} 6-\mathrm{C} 5$ & $-176.65(17)$ \\
\hline $\mathrm{C} 1-\mathrm{C} 2-\mathrm{C} 3-\mathrm{C} 7$ & $-177.19(18)$ & $\mathrm{C} 2-\mathrm{C} 3-\mathrm{C} 7-\mathrm{O} 1$ & $4.6(3)$ \\
\hline $\mathrm{C} 8-\mathrm{O} 2-\mathrm{C} 4-\mathrm{C} 5$ & $83.8(2)$ & $\mathrm{C} 4-\mathrm{C} 3-\mathrm{C} 7-\mathrm{O} 1$ & $-173.27(18)$ \\
\hline $\mathrm{C} 8-\mathrm{O} 2-\mathrm{C} 4-\mathrm{C} 3$ & $-101.3(2)$ & $\mathrm{C} 6-\mathrm{C} 5-\mathrm{C} 9-\mathrm{O} 3$ & $-22.3(3)$ \\
\hline $\mathrm{C} 2-\mathrm{C} 3-\mathrm{C} 4-\mathrm{O} 2$ & $-174.23(16)$ & $\mathrm{C} 4-\mathrm{C} 5-\mathrm{C} 9-\mathrm{O} 3$ & $155.81(18)$ \\
\hline $\mathrm{C} 7-\mathrm{C} 3-\mathrm{C} 4-\mathrm{O} 2$ & $3.7(3)$ & $\mathrm{C} 11-\mathrm{O} 5-\mathrm{C} 10-\mathrm{O} 4$ & $-3.3(3)$ \\
\hline $\mathrm{C} 2-\mathrm{C} 3-\mathrm{C} 4-\mathrm{C} 5$ & $0.6(3)$ & $\mathrm{C} 11-\mathrm{O} 5-\mathrm{C} 10-\mathrm{C} 1$ & $176.13(17)$ \\
\hline $\mathrm{C} 7-\mathrm{C} 3-\mathrm{C} 4-\mathrm{C} 5$ & $178.51(18)$ & $\mathrm{C} 6-\mathrm{C} 1-\mathrm{C} 10-\mathrm{O} 4$ & $172.68(19)$ \\
\hline $\mathrm{O} 2-\mathrm{C} 4-\mathrm{C} 5-\mathrm{C} 6$ & $173.67(17)$ & $\mathrm{C} 2-\mathrm{C} 1-\mathrm{C} 10-\mathrm{O} 4$ & $-5.1(3)$ \\
\hline $\mathrm{C} 3-\mathrm{C} 4-\mathrm{C} 5-\mathrm{C} 6$ & $-1.0(3)$ & $\mathrm{C} 6-\mathrm{C} 1-\mathrm{C} 10-\mathrm{O} 5$ & $-6.7(3)$ \\
\hline $\mathrm{O} 2-\mathrm{C} 4-\mathrm{C} 5-\mathrm{C} 9$ & $-4.5(3)$ & $\mathrm{C} 2-\mathrm{C} 1-\mathrm{C} 10-\mathrm{O} 5$ & $175.55(17)$ \\
\hline $\mathrm{C} 3-\mathrm{C} 4-\mathrm{C} 5-\mathrm{C} 9$ & $-179.19(18)$ & $\mathrm{C} 10-\mathrm{O} 5-\mathrm{C} 11-\mathrm{C} 12$ & $-93.5(3)$ \\
\hline $\mathrm{C} 4-\mathrm{C} 5-\mathrm{C} 6-\mathrm{C} 1$ & $0.2(3)$ & & \\
\hline
\end{tabular}

Hydrogen-bond geometry $\left(\AA,{ }^{o}\right)$ for $(I)$

\begin{tabular}{lllll}
\hline$D-\mathrm{H}^{\cdots} A$ & $D-\mathrm{H}$ & $\mathrm{H} \cdots A$ & $D \cdots A$ & $D-\mathrm{H} \cdots A$ \\
\hline $\mathrm{O} 1-\mathrm{H} 1 O \cdots \mathrm{O} 3^{\mathrm{i}}$ & $0.92(3)$ & $1.87(3)$ & $2.788(2)$ & $176(3)$ \\
$\mathrm{O} 3-\mathrm{H} 3 O \cdots{ }^{\mathrm{ii}}$ & $0.91(3)$ & $1.83(3)$ & $2.725(2)$ & $167(3)$ \\
$\mathrm{C} 9-\mathrm{H} 9 B \cdots \mathrm{O} 3^{\mathrm{ii}}$ & 0.99 & 2.65 & $3.555(3)$ & 153 \\
\hline
\end{tabular}

Symmetry codes: (i) $-x+1,-y+1,-z+1$; (ii) $x-1, y+1, z$; (iii) $-x,-y+2,-z+1$.

(II)

\section{Crystal data}

$\mathrm{C}_{9} \mathrm{H}_{11} \mathrm{BrO}_{3}$

$M_{r}=247.09$

Orthorhombic, Pna $2_{1}$

$a=13.1596$ (7) $\AA$

$b=16.2514(9) \AA$

$c=4.4735$ (2) $\AA$

$V=956.71(9) \AA^{3}$

$Z=4$

$F(000)=496$
$D_{\mathrm{x}}=1.715 \mathrm{Mg} \mathrm{m}^{-3}$

Melting point: $400.15 \mathrm{~K}$

Mo $K \alpha$ radiation, $\lambda=0.71073 \AA$

Cell parameters from 5411 reflections

$\theta=2.0-27.2^{\circ}$

$\mu=4.27 \mathrm{~mm}^{-1}$

$T=173 \mathrm{~K}$

Piece, colorless

$0.45 \times 0.27 \times 0.15 \mathrm{~mm}$ 


\section{Data collection}

STOE IPDS 2

diffractometer

Radiation source: sealed X-ray tube, 12 x $0.4 \mathrm{~mm}$

long-fine focus

Plane graphite monochromator

Detector resolution: 6.67 pixels $\mathrm{mm}^{-1}$

rotation method scans

Absorption correction: integration $X$-AREA (STOE)

\section{Refinement}

Refinement on $F^{2}$

Least-squares matrix: full

$R\left[F^{2}>2 \sigma\left(F^{2}\right)\right]=0.065$

$w R\left(F^{2}\right)=0.190$

$S=1.10$

1904 reflections

127 parameters

1 restraint

Hydrogen site location: mixed
$T_{\min }=0.283, T_{\max }=0.564$

4183 measured reflections

1904 independent reflections

1647 reflections with $I>2 \sigma(I)$

$R_{\text {int }}=0.074$

$\theta_{\max }=26.5^{\circ}, \theta_{\min }=2.5^{\circ}$

$h=-13 \rightarrow 16$

$k=-20 \rightarrow 17$

$l=-5 \rightarrow 5$

$\mathrm{H}$ atoms treated by a mixture of independent and constrained refinement

$w=1 /\left[\sigma^{2}\left(F_{\mathrm{o}}^{2}\right)+(0.123 P)^{2}+3.1968 P\right]$

where $P=\left(F_{\mathrm{o}}^{2}+2 F_{\mathrm{c}}{ }^{2}\right) / 3$

$(\Delta / \sigma)_{\max }<0.001$

$\Delta \rho_{\max }=0.82 \mathrm{e} \AA^{-3}$

$\Delta \rho_{\min }=-1.22 \mathrm{e} \AA^{-3}$

Absolute structure: Classical Flack method preferred over Parsons because s.u. lower.

Absolute structure parameter: 0.01 (5)

\section{Special details}

Geometry. All esds (except the esd in the dihedral angle between two 1.s. planes) are estimated using the full covariance matrix. The cell esds are taken into account individually in the estimation of esds in distances, angles and torsion angles; correlations between esds in cell parameters are only used when they are defined by crystal symmetry. An approximate (isotropic) treatment of cell esds is used for estimating esds involving 1.s. planes.

Refinement. $\mathrm{H}$ atoms were positioned geometrically and allowed to ride on their parent atoms, $\mathrm{C}-\mathrm{H}=0.95-0.98 \% \mathrm{~A}$ and $\mathrm{Uiso}(\mathrm{H})=$ 1.2-1.5 Ueq (parent atom). Hydrogen atoms bonded to $\mathrm{O}$ were located via electron density. Individual reflections were considered as outliers and therefore omitted.

Fractional atomic coordinates and isotropic or equivalent isotropic displacement parameters $\left(\AA^{2}\right)$ for (II)

\begin{tabular}{lllll}
\hline & $x$ & $y$ & $z$ & $U_{\text {iso }} * / U_{\text {eq }}$ \\
\hline Br1 & $0.47945(7)$ & $0.58610(5)$ & $0.1742(5)$ & $0.0274(4)$ \\
O1 & $0.1690(7)$ & $0.6471(6)$ & $0.924(2)$ & $0.0309(18)$ \\
O2 & $0.2989(7)$ & $0.8804(5)$ & $0.7961(19)$ & $0.0284(18)$ \\
O3 & $0.5787(7)$ & $0.9079(5)$ & $0.424(2)$ & $0.0248(17)$ \\
C1 & $0.4222(8)$ & $0.6800(6)$ & $0.371(2)$ & $0.019(2)$ \\
C2 & $0.3384(9)$ & $0.6688(7)$ & $0.554(3)$ & $0.023(2)$ \\
H2 & 0.3111 & 0.6155 & 0.5870 & $0.028^{*}$ \\
C3 & $0.2954(7)$ & $0.7370(6)$ & $0.689(4)$ & $0.0215(19)$ \\
C4 & $0.3399(8)$ & $0.8139(6)$ & $0.640(3)$ & $0.019(2)$ \\
C5 & $0.4253(8)$ & $0.8240(6)$ & $0.462(2)$ & $0.021(2)$ \\
C6 & $0.4652(8)$ & $0.7551(7)$ & $0.321(3)$ & $0.023(2)$ \\
H6 & 0.5220 & 0.7602 & 0.1915 & $0.027^{*}$ \\
C7 & $0.2037(9)$ & $0.7300(8)$ & $0.889(3)$ & $0.027(2)$ \\
H7A & 0.2206 & 0.7529 & 1.0878 & $0.032^{*}$ \\
H7B & 0.1479 & 0.7636 & 0.8040 & $0.032^{*}$ \\
C8 & $0.2333(12)$ & $0.9311(9)$ & $0.627(5)$ & $0.049(5)$ \\
H8A & 0.2158 & 0.9801 & 0.7438 & $0.073^{*}$ \\
H8B & 0.1713 & 0.9005 & 0.5787 & $0.073^{*}$ \\
H8C & 0.2674 & 0.9477 & 0.4420 & $0.073^{*}$
\end{tabular}




\begin{tabular}{|c|c|c|c|c|}
\hline C9 & $0.4713(10)$ & $0.9082(6)$ & $0.411(4)$ & $0.029(3)$ \\
\hline H9A & 0.4498 & 0.9286 & 0.2120 & $0.035^{*}$ \\
\hline H9B & 0.4447 & 0.9467 & 0.5632 & $0.035^{*}$ \\
\hline $\mathrm{H} 1$ & $0.13(2)$ & $0.608(14)$ & $0.79(5)$ & $0.10(9)^{*}$ \\
\hline $\mathrm{H} 3$ & $0.591(14)$ & $0.888(10)$ & $0.27(4)$ & $0.04(5)^{*}$ \\
\hline
\end{tabular}

Atomic displacement parameters $\left(\AA^{2}\right)$ for (II)

\begin{tabular}{lllllll}
\hline & $U^{11}$ & $U^{22}$ & $U^{33}$ & $U^{12}$ & $U^{13}$ & $U^{23}$ \\
\hline $\mathrm{Br} 1$ & $0.0282(6)$ & $0.0184(5)$ & $0.0357(6)$ & $0.0039(3)$ & $0.0029(8)$ & $-0.0012(7)$ \\
$\mathrm{O} 1$ & $0.029(4)$ & $0.040(5)$ & $0.024(4)$ & $-0.012(4)$ & $0.008(3)$ & $0.004(4)$ \\
$\mathrm{O} 2$ & $0.030(4)$ & $0.025(4)$ & $0.030(4)$ & $0.004(4)$ & $0.002(3)$ & $-0.011(3)$ \\
$\mathrm{O} 3$ & $0.021(4)$ & $0.022(4)$ & $0.031(5)$ & $-0.003(3)$ & $0.003(3)$ & $-0.004(3)$ \\
$\mathrm{C} 1$ & $0.014(5)$ & $0.020(5)$ & $0.022(5)$ & $-0.005(4)$ & $0.000(4)$ & $0.000(4)$ \\
C2 & $0.029(6)$ & $0.022(5)$ & $0.020(4)$ & $-0.007(4)$ & $-0.002(4)$ & $0.005(4)$ \\
C3 & $0.021(4)$ & $0.022(4)$ & $0.021(5)$ & $0.001(3)$ & $-0.001(7)$ & $-0.001(5)$ \\
C4 & $0.020(4)$ & $0.023(4)$ & $0.015(5)$ & $0.009(4)$ & $0.001(4)$ & $-0.005(4)$ \\
C5 & $0.020(5)$ & $0.016(5)$ & $0.026(5)$ & $0.001(4)$ & $-0.002(4)$ & $0.001(4)$ \\
C6 & $0.019(5)$ & $0.023(5)$ & $0.026(6)$ & $0.006(4)$ & $0.005(4)$ & $0.003(5)$ \\
C7 & $0.021(5)$ & $0.037(6)$ & $0.023(6)$ & $-0.004(5)$ & $0.006(4)$ & $0.004(4)$ \\
C8 & $0.035(7)$ & $0.038(6)$ & $0.074(15)$ & $0.017(6)$ & $0.002(8)$ & $-0.005(8)$ \\
C9 & $0.032(8)$ & $0.010(5)$ & $0.046(8)$ & $-0.002(4)$ & $0.001(5)$ & $0.000(4)$
\end{tabular}

Geometric parameters (A, $\left.{ }^{\circ}\right)$ for (II)

\begin{tabular}{|c|c|c|c|}
\hline $\mathrm{Br} 1-\mathrm{C} 1$ & $1.916(11)$ & $\mathrm{C} 3-\mathrm{C} 7$ & $1.506(16)$ \\
\hline $\mathrm{O} 1-\mathrm{C} 7$ & $1.431(15)$ & $\mathrm{C} 4-\mathrm{C} 5$ & $1.387(15)$ \\
\hline $\mathrm{O} 1-\mathrm{H} 1$ & $1.0(2)$ & $\mathrm{C} 5-\mathrm{C} 6$ & $1.388(15)$ \\
\hline $\mathrm{O} 2-\mathrm{C} 4$ & $1.396(13)$ & $\mathrm{C} 5-\mathrm{C} 9$ & $1.513(14)$ \\
\hline $\mathrm{O} 2-\mathrm{C} 8$ & $1.411(19)$ & $\mathrm{C} 6-\mathrm{H} 6$ & 0.9500 \\
\hline $\mathrm{O} 3-\mathrm{C} 9$ & $1.415(17)$ & $\mathrm{C} 7-\mathrm{H} 7 \mathrm{~A}$ & 0.9900 \\
\hline $\mathrm{O} 3-\mathrm{H} 3$ & $0.76(16)$ & $\mathrm{C} 7-\mathrm{H} 7 \mathrm{~B}$ & 0.9900 \\
\hline $\mathrm{C} 1-\mathrm{C} 6$ & $1.364(15)$ & $\mathrm{C} 8-\mathrm{H} 8 \mathrm{~A}$ & 0.9800 \\
\hline $\mathrm{C} 1-\mathrm{C} 2$ & $1.387(15)$ & $\mathrm{C} 8-\mathrm{H} 8 \mathrm{~B}$ & 0.9800 \\
\hline $\mathrm{C} 2-\mathrm{C} 3$ & $1.382(17)$ & $\mathrm{C} 8-\mathrm{H} 8 \mathrm{C}$ & 0.9800 \\
\hline $\mathrm{C} 2-\mathrm{H} 2$ & 0.9500 & C9- $\mathrm{H} 9 \mathrm{~A}$ & 0.9900 \\
\hline $\mathrm{C} 3-\mathrm{C} 4$ & $1.397(14)$ & C9-H9B & 0.9900 \\
\hline $\mathrm{C} 7-\mathrm{O} 1-\mathrm{H} 1$ & $132(10)$ & $\mathrm{C} 5-\mathrm{C} 6-\mathrm{H} 6$ & 120.3 \\
\hline $\mathrm{C} 4-\mathrm{O} 2-\mathrm{C} 8$ & $114.8(11)$ & $\mathrm{O} 1-\mathrm{C} 7-\mathrm{C} 3$ & $113.1(9)$ \\
\hline $\mathrm{C} 9-\mathrm{O} 3-\mathrm{H} 3$ & $101(10)$ & $\mathrm{O} 1-\mathrm{C} 7-\mathrm{H} 7 \mathrm{~A}$ & 109.0 \\
\hline $\mathrm{C} 6-\mathrm{C} 1-\mathrm{C} 2$ & $123.0(10)$ & $\mathrm{C} 3-\mathrm{C} 7-\mathrm{H} 7 \mathrm{~A}$ & 109.0 \\
\hline $\mathrm{C} 6-\mathrm{C} 1-\mathrm{Br} 1$ & $118.3(8)$ & $\mathrm{O} 1-\mathrm{C} 7-\mathrm{H} 7 \mathrm{~B}$ & 109.0 \\
\hline $\mathrm{C} 2-\mathrm{C} 1-\mathrm{Br} 1$ & $118.7(8)$ & $\mathrm{C} 3-\mathrm{C} 7-\mathrm{H} 7 \mathrm{~B}$ & 109.0 \\
\hline $\mathrm{C} 3-\mathrm{C} 2-\mathrm{C} 1$ & $118.6(10)$ & $\mathrm{H} 7 \mathrm{~A}-\mathrm{C} 7-\mathrm{H} 7 \mathrm{~B}$ & 107.8 \\
\hline $\mathrm{C} 3-\mathrm{C} 2-\mathrm{H} 2$ & 120.7 & $\mathrm{O} 2-\mathrm{C} 8-\mathrm{H} 8 \mathrm{~A}$ & 109.5 \\
\hline $\mathrm{C} 1-\mathrm{C} 2-\mathrm{H} 2$ & 120.7 & $\mathrm{O} 2-\mathrm{C} 8-\mathrm{H} 8 \mathrm{~B}$ & 109.5 \\
\hline $\mathrm{C} 2-\mathrm{C} 3-\mathrm{C} 4$ & $118.5(11)$ & $\mathrm{H} 8 \mathrm{~A}-\mathrm{C} 8-\mathrm{H} 8 \mathrm{~B}$ & 109.5 \\
\hline $\mathrm{C} 2-\mathrm{C} 3-\mathrm{C} 7$ & $121.7(9)$ & $\mathrm{O} 2-\mathrm{C} 8-\mathrm{H} 8 \mathrm{C}$ & 109.5 \\
\hline $\mathrm{C} 4-\mathrm{C} 3-\mathrm{C} 7$ & $119.8(10)$ & $\mathrm{H} 8 \mathrm{~A}-\mathrm{C} 8-\mathrm{H} 8 \mathrm{C}$ & 109.5 \\
\hline $\mathrm{C} 5-\mathrm{C} 4-\mathrm{O} 2$ & $120.6(9)$ & $\mathrm{H} 8 \mathrm{~B}-\mathrm{C} 8-\mathrm{H} 8 \mathrm{C}$ & 109.5 \\
\hline
\end{tabular}




\begin{tabular}{|c|c|c|c|}
\hline $\mathrm{C} 5-\mathrm{C} 4-\mathrm{C} 3$ & $122.4(9)$ & $\mathrm{O} 3-\mathrm{C} 9-\mathrm{C} 5$ & $113.0(9)$ \\
\hline $\mathrm{O} 2-\mathrm{C} 4-\mathrm{C} 3$ & $116.9(10)$ & $\mathrm{O} 3-\mathrm{C} 9-\mathrm{H} 9 \mathrm{~A}$ & 109.0 \\
\hline $\mathrm{C} 4-\mathrm{C} 5-\mathrm{C} 6$ & $118.1(10)$ & $\mathrm{C} 5-\mathrm{C} 9-\mathrm{H} 9 \mathrm{~A}$ & 109.0 \\
\hline $\mathrm{C} 4-\mathrm{C} 5-\mathrm{C} 9$ & $121.2(10)$ & $\mathrm{O} 3-\mathrm{C} 9-\mathrm{H} 9 \mathrm{~B}$ & 109.0 \\
\hline $\mathrm{C} 6-\mathrm{C} 5-\mathrm{C} 9$ & $120.6(11)$ & $\mathrm{C} 5-\mathrm{C} 9-\mathrm{H} 9 \mathrm{~B}$ & 109.0 \\
\hline $\mathrm{C} 1-\mathrm{C} 6-\mathrm{C} 5$ & $119.4(10)$ & $\mathrm{H} 9 \mathrm{~A}-\mathrm{C} 9-\mathrm{H} 9 \mathrm{~B}$ & 107.8 \\
\hline $\mathrm{C} 1-\mathrm{C} 6-\mathrm{H} 6$ & 120.3 & & \\
\hline $\mathrm{C} 6-\mathrm{C} 1-\mathrm{C} 2-\mathrm{C} 3$ & $1.2(17)$ & $\mathrm{C} 2-\mathrm{C} 1-\mathrm{C} 6-\mathrm{C} 5$ & $0.9(17)$ \\
\hline $\mathrm{Br} 1-\mathrm{C} 1-\mathrm{C} 2-\mathrm{C} 3$ & $-178.1(10)$ & $\mathrm{Br} 1-\mathrm{C} 1-\mathrm{C} 6-\mathrm{C} 5$ & $-179.8(8)$ \\
\hline $\mathrm{C} 1-\mathrm{C} 2-\mathrm{C} 3-\mathrm{C} 4$ & $-1.6(18)$ & $\mathrm{C} 4-\mathrm{C} 5-\mathrm{C} 6-\mathrm{C} 1$ & $-2.5(17)$ \\
\hline $\mathrm{C} 1-\mathrm{C} 2-\mathrm{C} 3-\mathrm{C} 7$ & $179.4(11)$ & $\mathrm{C} 9-\mathrm{C} 5-\mathrm{C} 6-\mathrm{C} 1$ & $-179.9(11)$ \\
\hline $\mathrm{C} 8-\mathrm{O} 2-\mathrm{C} 4-\mathrm{C} 5$ & $83.0(14)$ & $\mathrm{C} 2-\mathrm{C} 3-\mathrm{C} 7-\mathrm{O} 1$ & $0.9(17)$ \\
\hline $\mathrm{C} 8-\mathrm{O} 2-\mathrm{C} 4-\mathrm{C} 3$ & $-101.3(13)$ & $\mathrm{C} 4-\mathrm{C} 3-\mathrm{C} 7-\mathrm{O} 1$ & $-178.1(12)$ \\
\hline $\mathrm{C} 2-\mathrm{C} 3-\mathrm{C} 4-\mathrm{C} 5$ & $0.0(19)$ & $\mathrm{C} 4-\mathrm{C} 5-\mathrm{C} 9-\mathrm{O} 3$ & $137.6(12)$ \\
\hline $\mathrm{C} 7-\mathrm{C} 3-\mathrm{C} 4-\mathrm{C} 5$ & $179.0(11)$ & $\mathrm{C} 6-\mathrm{C} 5-\mathrm{C} 9-\mathrm{O} 3$ & $-45.0(17)$ \\
\hline $\mathrm{C} 2-\mathrm{C} 3-\mathrm{C} 4-\mathrm{O} 2$ & $-175.6(11)$ & $\mathrm{C} 2-\mathrm{C} 3-\mathrm{C} 7-\mathrm{O} 1$ & $0.9(17)$ \\
\hline $\mathrm{C} 7-\mathrm{C} 3-\mathrm{C} 4-\mathrm{O} 2$ & $3.5(17)$ & $\mathrm{C} 4-\mathrm{C} 3-\mathrm{C} 7-\mathrm{O} 1$ & $-178.1(12)$ \\
\hline $\mathrm{O} 2-\mathrm{C} 4-\mathrm{C} 5-\mathrm{C} 6$ & $177.5(10)$ & $\mathrm{C} 6-\mathrm{C} 5-\mathrm{C} 9-\mathrm{O} 3$ & $-45.0(17)$ \\
\hline $\mathrm{C} 3-\mathrm{C} 4-\mathrm{C} 5-\mathrm{C} 6$ & $2.1(18)$ & $\mathrm{C} 4-\mathrm{C} 5-\mathrm{C} 9-\mathrm{O} 3$ & $137.6(12)$ \\
\hline $\mathrm{O} 2-\mathrm{C} 4-\mathrm{C} 5-\mathrm{C} 9$ & $-5.1(17)$ & $\mathrm{C} 3-\mathrm{C} 4-\mathrm{O} 2-\mathrm{C} 8$ & $-101.3(13)$ \\
\hline $\mathrm{C} 3-\mathrm{C} 4-\mathrm{C} 5-\mathrm{C} 9$ & $179.5(12)$ & $\mathrm{C} 5-\mathrm{C} 4-\mathrm{O} 2-\mathrm{C} 8$ & $83.0(14)$ \\
\hline
\end{tabular}

Hydrogen-bond geometry $\left(\AA,{ }^{\circ}\right)$ for (II)

\begin{tabular}{lllll}
\hline$D-\mathrm{H}^{*} \cdots A$ & $D-\mathrm{H}$ & $\mathrm{H} \cdots A$ & $D \cdots A$ & $D-\mathrm{H} \cdots A$ \\
\hline $\mathrm{C} 8-\mathrm{H} 8 A \cdots \mathrm{Br} 1^{\mathrm{i}}$ & 0.98 & 3.11 & $3.773(13)$ & 126 \\
$\mathrm{C} 8-\mathrm{H} 8 B \cdots \mathrm{Br}^{\mathrm{ii}}$ & 0.98 & 3.11 & $3.917(19)$ & 140 \\
$\mathrm{O} 1-\mathrm{H} 1 \cdots \mathrm{O} 3{ }^{\mathrm{ii}}$ & $1.0(2)$ & $1.8(2)$ & $2.687(13)$ & $146(20)$ \\
$\mathrm{O} 3-\mathrm{H} 3 \cdots 1^{\mathrm{iii}}$ & $0.76(16)$ & $1.95(17)$ & $2.685(13)$ & $160(18)$ \\
\hline
\end{tabular}

Symmetry codes: (i) $-x+1 / 2, y+1 / 2, z+1 / 2$; (ii) $x-1 / 2,-y+3 / 2, z$; (iii) $x+1 / 2,-y+3 / 2, z-1$.

\section{(III) 4-Bromo-2,6-bis(bromomethyl)anisole}

Crystal data

\section{$\mathrm{C}_{9} \mathrm{H}_{9} \mathrm{Br}_{3} \mathrm{O}$}

$M_{r}=372.89$

Monoclinic, $C 2 / c$

$a=33.315$ (4) $\AA$

$b=4.3913$ (3) $\AA$

$c=15.9655(17) \AA$

$\beta=110.142(8)^{\circ}$

$V=2192.9$ (4) $\AA^{3}$

$Z=8$

$F(000)=1408$

\section{Data collection}

STOE IPDS 2T

diffractometer

Radiation source: sealed X-ray tube, 12 x $0.4 \mathrm{~mm}$ long-fine focus

Plane graphite monochromator
$D_{\mathrm{x}}=2.259 \mathrm{Mg} \mathrm{m}^{-3}$

Melting point: $358.15 \mathrm{~K}$

Mo $K \alpha$ radiation, $\lambda=0.71073 \AA$

Cell parameters from 6186 reflections

$\theta=2.5-27.1^{\circ}$

$\mu=11.00 \mathrm{~mm}^{-1}$

$T=153 \mathrm{~K}$

Piece, colorless

$0.50 \times 0.11 \times 0.09 \mathrm{~mm}$

Detector resolution: 6.67 pixels $\mathrm{mm}^{-1}$ rotation method scans

Absorption correction: integration $X$-AREA (STOE)

$T_{\min }=0.008, T_{\max }=0.076$ 
5754 measured reflections

2197 independent reflections

1834 reflections with $I>2 \sigma(I)$

$R_{\text {int }}=0.087$

Refinement

Refinement on $F^{2}$

Least-squares matrix: full

$R\left[F^{2}>2 \sigma\left(F^{2}\right)\right]=0.033$

$w R\left(F^{2}\right)=0.088$

$S=1.10$

2197 reflections

119 parameters

0 restraints

$$
\begin{aligned}
& \theta_{\max }=26.2^{\circ}, \theta_{\min }=2.6^{\circ} \\
& h=-33 \rightarrow 40 \\
& k=-5 \rightarrow 4 \\
& l=-19 \rightarrow 19
\end{aligned}
$$

Hydrogen site location: inferred from neighbouring sites

$\mathrm{H}$-atom parameters constrained

$w=1 /\left[\sigma^{2}\left(F_{\mathrm{o}}^{2}\right)+(0.0412 P)^{2}+2.9694 P\right]$

$$
\text { where } P=\left(F_{\mathrm{o}}{ }^{2}+2 F_{\mathrm{c}}{ }^{2}\right) / 3
$$

$(\Delta / \sigma)_{\max }=0.001$

$\Delta \rho_{\max }=0.63$ e $\AA^{-3}$

$\Delta \rho_{\min }=-0.60 \mathrm{e} \AA^{-3}$

\section{Special details}

Geometry. All esds (except the esd in the dihedral angle between two 1.s. planes) are estimated using the full covariance matrix. The cell esds are taken into account individually in the estimation of esds in distances, angles and torsion angles; correlations between esds in cell parameters are only used when they are defined by crystal symmetry. An approximate (isotropic) treatment of cell esds is used

\begin{tabular}{|c|c|c|c|c|}
\hline & $x$ & $y$ & $z$ & $U_{\text {iso }} * / U_{\text {eq }}$ \\
\hline Br1 & 0.47987 (2) & $0.49550(12)$ & 0.35631 & $0.03202(15)$ \\
\hline $\mathrm{Br} 2$ & $0.29357(2)$ & $0.25013(12)$ & 0.15688 & $0.03636(16)$ \\
\hline $\mathrm{Br} 3$ & $0.42282(2)$ & $0.06192(11)$ & $0.65117(3)$ & $0.03003(14)$ \\
\hline $\mathrm{O} 1$ & $0.32003(9)$ & $0.0508(7)$ & $0.4161(2)$ & $0.0277(7)$ \\
\hline $\mathrm{C} 1$ & $0.42990(12)$ & $0.3516(10)$ & $0.3754(3)$ & $0.0221(8)$ \\
\hline $\mathrm{C} 2$ & $0.39000(13)$ & $0.4533(10)$ & $0.3190(3)$ & $0.0244(9)$ \\
\hline $\mathrm{H} 2$ & 0.3880 & 0.5895 & 0.2715 & $0.029 *$ \\
\hline $\mathrm{C} 3$ & $0.35315(12)$ & $0.3527(10)$ & $0.3331(3)$ & $0.0205(8)$ \\
\hline $\mathrm{C} 4$ & $0.35679(12)$ & $0.1583(9)$ & $0.4038(3)$ & $0.0195(8)$ \\
\hline $\mathrm{C} 5$ & $0.39681(13)$ & $0.0557(9)$ & $0.4607(3)$ & $0.0208(8)$ \\
\hline C6 & $0.43327(12)$ & $0.1556(10)$ & $0.4443(3)$ & $0.0199(8)$ \\
\hline H6 & 0.4607 & 0.0868 & 0.4813 & $0.024 *$ \\
\hline $\mathrm{C} 7$ & 0.31004 (14) & $0.4628(11)$ & $0.2722(3)$ & $0.0295(10)$ \\
\hline H7A & 0.3112 & 0.6848 & 0.2625 & $0.035^{*}$ \\
\hline H7B & 0.2883 & 0.4250 & 0.3004 & $0.035^{*}$ \\
\hline $\mathrm{C} 8$ & $0.30694(14)$ & $0.2317(11)$ & $0.4772(3)$ & $0.0296(10)$ \\
\hline H8A & 0.2805 & 0.1481 & 0.4817 & $0.044^{*}$ \\
\hline H8B & 0.3020 & 0.4419 & 0.4555 & $0.044 *$ \\
\hline $\mathrm{H} 8 \mathrm{C}$ & 0.3294 & 0.2284 & 0.5361 & $0.044 *$ \\
\hline C9 & $0.40141(14)$ & $-0.1557(11)$ & $0.5363(3)$ & $0.0273(9)$ \\
\hline H9A & 0.4215 & -0.3212 & 0.5359 & $0.033^{*}$ \\
\hline H9B & 0.3733 & -0.2488 & 0.5291 & $0.033^{*}$ \\
\hline
\end{tabular}
for estimating esds involving 1.s. planes.

Refinement. $\mathrm{H}$ atoms were positioned geometrically and allowed to ride on their parent atoms, $\mathrm{C}-\mathrm{H}=0.95-0.98 \% \mathrm{~A}$ and $\mathrm{Uiso}(\mathrm{H})=$ 1.2-1.5 Ueq (parent atom).

Fractional atomic coordinates and isotropic or equivalent isotropic displacement parameters $\left(\AA^{2}\right)$ for (III)

Atomic displacement parameters $\left(\AA^{2}\right)$ for (III)

\begin{tabular}{lllllll}
\hline & $U^{11}$ & $U^{22}$ & $U^{33}$ & $U^{12}$ & $U^{13}$ & $U^{23}$ \\
\hline $\operatorname{Br} 1$ & $0.0210(2)$ & $0.0455(3)$ & $0.0322(3)$ & $-0.00607(19)$ & $0.01262(19)$ & $-0.0037(2)$
\end{tabular}




$\begin{array}{lllllll}\text { Br2 } & 0.0317(2) & 0.0407(3) & 0.0278(3) & 0.0014(2) & -0.00112(19) & -0.0017(2) \\ \text { Br3 } & 0.0344(2) & 0.0322(2) & 0.0236(3) & 0.00105(19) & 0.01019(19) & 0.00098(19) \\ \text { O1 } & 0.0219(14) & 0.0262(15) & 0.0382(18) & -0.0063(13) & 0.0143(14) & -0.0061(15) \\ \text { C1 } & 0.0188(18) & 0.026(2) & 0.022(2) & -0.0070(17) & 0.0084(16) & -0.0090(19) \\ \text { C2 } & 0.023(2) & 0.026(2) & 0.024(2) & -0.0003(17) & 0.0084(17) & -0.0005(18) \\ \text { C3 } & 0.0182(17) & 0.0199(19) & 0.022(2) & -0.0008(17) & 0.0051(15) & -0.0056(18) \\ \text { C4 } & 0.0204(18) & 0.0167(17) & 0.024(2) & -0.0038(16) & 0.0106(16) & -0.0088(18) \\ \text { C5 } & 0.025(2) & 0.0151(18) & 0.024(2) & -0.0018(16) & 0.0107(17) & -0.0072(17) \\ \text { C6 } & 0.0143(16) & 0.025(2) & 0.0174(18) & 0.0029(17) & 0.0021(14) & -0.0061(18) \\ \text { C7 } & 0.023(2) & 0.030(2) & 0.030(2) & 0.0078(19) & 0.0033(19) & 0.000(2) \\ \text { C8 } & 0.023(2) & 0.035(3) & 0.035(2) & 0.0003(19) & 0.0148(19) & 0.000(2) \\ \text { C9 } & 0.031(2) & 0.021(2) & 0.028(2) & -0.0012(19) & 0.0081(18) & -0.001(2)\end{array}$

Geometric parameters $\left(\AA,{ }^{\circ}\right)$ for (III)

\begin{tabular}{|c|c|c|c|}
\hline $\mathrm{Br} 1-\mathrm{C} 1$ & $1.901(4)$ & $\mathrm{C} 4-\mathrm{C} 5$ & $1.404(6)$ \\
\hline $\mathrm{Br} 2-\mathrm{C} 7$ & $1.966(5)$ & $\mathrm{C} 5-\mathrm{C} 6$ & $1.399(6)$ \\
\hline $\mathrm{Br} 3-\mathrm{C} 9$ & $1.971(4)$ & $\mathrm{C} 5-\mathrm{C} 9$ & $1.488(6)$ \\
\hline $\mathrm{O} 1-\mathrm{C} 4$ & $1.387(5)$ & C6- $\mathrm{H} 6$ & 0.9500 \\
\hline $\mathrm{O} 1-\mathrm{C} 8$ & $1.437(5)$ & $\mathrm{C} 7-\mathrm{H} 7 \mathrm{~A}$ & 0.9900 \\
\hline $\mathrm{C} 1-\mathrm{C} 6$ & $1.370(6)$ & $\mathrm{C} 7-\mathrm{H} 7 \mathrm{~B}$ & 0.9900 \\
\hline $\mathrm{C} 1-\mathrm{C} 2$ & $1.398(6)$ & $\mathrm{C} 8-\mathrm{H} 8 \mathrm{~A}$ & 0.9800 \\
\hline $\mathrm{C} 2-\mathrm{C} 3$ & $1.394(6)$ & $\mathrm{C} 8-\mathrm{H} 8 \mathrm{~B}$ & 0.9800 \\
\hline $\mathrm{C} 2-\mathrm{H} 2$ & 0.9500 & $\mathrm{C} 8-\mathrm{H} 8 \mathrm{C}$ & 0.9800 \\
\hline $\mathrm{C} 3-\mathrm{C} 4$ & $1.387(6)$ & $\mathrm{C} 9-\mathrm{H} 9 \mathrm{~A}$ & 0.9900 \\
\hline $\mathrm{C} 3-\mathrm{C} 7$ & $1.510(6)$ & $\mathrm{C} 9-\mathrm{H} 9 \mathrm{~B}$ & 0.9900 \\
\hline $\mathrm{C} 4-\mathrm{O} 1-\mathrm{C} 8$ & $113.4(3)$ & $\mathrm{C} 3-\mathrm{C} 7-\mathrm{Br} 2$ & $110.4(3)$ \\
\hline $\mathrm{C} 6-\mathrm{C} 1-\mathrm{C} 2$ & $121.0(4)$ & $\mathrm{C} 3-\mathrm{C} 7-\mathrm{H} 7 \mathrm{~A}$ & 109.6 \\
\hline $\mathrm{C} 6-\mathrm{C} 1-\mathrm{Br} 1$ & $120.2(3)$ & $\mathrm{Br} 2-\mathrm{C} 7-\mathrm{H} 7 \mathrm{~A}$ & 109.6 \\
\hline $\mathrm{C} 2-\mathrm{C} 1-\mathrm{Br} 1$ & $118.8(3)$ & $\mathrm{C} 3-\mathrm{C} 7-\mathrm{H} 7 \mathrm{~B}$ & 109.6 \\
\hline $\mathrm{C} 3-\mathrm{C} 2-\mathrm{C} 1$ & $119.3(4)$ & $\mathrm{Br} 2-\mathrm{C} 7-\mathrm{H} 7 \mathrm{~B}$ & 109.6 \\
\hline $\mathrm{C} 3-\mathrm{C} 2-\mathrm{H} 2$ & 120.3 & $\mathrm{H} 7 \mathrm{~A}-\mathrm{C} 7-\mathrm{H} 7 \mathrm{~B}$ & 108.1 \\
\hline $\mathrm{C} 1-\mathrm{C} 2-\mathrm{H} 2$ & 120.3 & $\mathrm{O} 1-\mathrm{C} 8-\mathrm{H} 8 \mathrm{~A}$ & 109.5 \\
\hline $\mathrm{C} 4-\mathrm{C} 3-\mathrm{C} 2$ & $119.4(4)$ & $\mathrm{O} 1-\mathrm{C} 8-\mathrm{H} 8 \mathrm{~B}$ & 109.5 \\
\hline $\mathrm{C} 4-\mathrm{C} 3-\mathrm{C} 7$ & $121.2(4)$ & $\mathrm{H} 8 \mathrm{~A}-\mathrm{C} 8-\mathrm{H} 8 \mathrm{~B}$ & 109.5 \\
\hline $\mathrm{C} 2-\mathrm{C} 3-\mathrm{C} 7$ & $119.3(4)$ & $\mathrm{O} 1-\mathrm{C} 8-\mathrm{H} 8 \mathrm{C}$ & 109.5 \\
\hline $\mathrm{C} 3-\mathrm{C} 4-\mathrm{O} 1$ & $119.3(3)$ & $\mathrm{H} 8 \mathrm{~A}-\mathrm{C} 8-\mathrm{H} 8 \mathrm{C}$ & 109.5 \\
\hline $\mathrm{C} 3-\mathrm{C} 4-\mathrm{C} 5$ & $121.4(4)$ & $\mathrm{H} 8 \mathrm{~B}-\mathrm{C} 8-\mathrm{H} 8 \mathrm{C}$ & 109.5 \\
\hline $\mathrm{O} 1-\mathrm{C} 4-\mathrm{C} 5$ & $119.2(4)$ & $\mathrm{C} 5-\mathrm{C} 9-\mathrm{Br} 3$ & $110.8(3)$ \\
\hline $\mathrm{C} 6-\mathrm{C} 5-\mathrm{C} 4$ & $118.1(4)$ & $\mathrm{C} 5-\mathrm{C} 9-\mathrm{H} 9 \mathrm{~A}$ & 109.5 \\
\hline $\mathrm{C} 6-\mathrm{C} 5-\mathrm{C} 9$ & $119.6(4)$ & $\mathrm{Br} 3-\mathrm{C} 9-\mathrm{H} 9 \mathrm{~A}$ & 109.5 \\
\hline $\mathrm{C} 4-\mathrm{C} 5-\mathrm{C} 9$ & $122.3(4)$ & $\mathrm{C} 5-\mathrm{C} 9-\mathrm{H} 9 \mathrm{~B}$ & 109.5 \\
\hline $\mathrm{C} 1-\mathrm{C} 6-\mathrm{C} 5$ & $120.7(4)$ & $\mathrm{Br} 3-\mathrm{C} 9-\mathrm{H} 9 \mathrm{~B}$ & 109.5 \\
\hline $\mathrm{C} 1-\mathrm{C} 6-\mathrm{H} 6$ & 119.6 & $\mathrm{H} 9 \mathrm{~A}-\mathrm{C} 9-\mathrm{H} 9 \mathrm{~B}$ & 108.1 \\
\hline $\mathrm{C} 5-\mathrm{C} 6-\mathrm{H} 6$ & 119.6 & & \\
\hline $\mathrm{C} 6-\mathrm{C} 1-\mathrm{C} 2-\mathrm{C} 3$ & $-0.1(7)$ & $\mathrm{O} 1-\mathrm{C} 4-\mathrm{C} 5-\mathrm{C} 6$ & $176.9(3)$ \\
\hline $\mathrm{Br} 1-\mathrm{C} 1-\mathrm{C} 2-\mathrm{C} 3$ & $-179.1(3)$ & $\mathrm{C} 3-\mathrm{C} 4-\mathrm{C} 5-\mathrm{C} 9$ & $-179.3(4)$ \\
\hline $\mathrm{C} 1-\mathrm{C} 2-\mathrm{C} 3-\mathrm{C} 4$ & $1.2(6)$ & $\mathrm{O} 1-\mathrm{C} 4-\mathrm{C} 5-\mathrm{C} 9$ & $-2.4(6)$ \\
\hline $\mathrm{C} 1-\mathrm{C} 2-\mathrm{C} 3-\mathrm{C} 7$ & $180.0(4)$ & $\mathrm{C} 2-\mathrm{C} 1-\mathrm{C} 6-\mathrm{C} 5$ & $-1.0(6)$ \\
\hline
\end{tabular}




$\begin{array}{ll}\mathrm{C} 2-\mathrm{C} 3-\mathrm{C} 4-\mathrm{O} 1 & -178.0(4) \\ \mathrm{C} 7-\mathrm{C} 3-\mathrm{C} 4-\mathrm{O} 1 & 3.3(6) \\ \mathrm{C} 2-\mathrm{C} 3-\mathrm{C} 4-\mathrm{C} 5 & -1.2(6) \\ \mathrm{C} 7-\mathrm{C} 3-\mathrm{C} 4-\mathrm{C} 5 & -179.9(4) \\ \mathrm{C} 8-\mathrm{O} 1-\mathrm{C} 4-\mathrm{C} 3 & -94.0(4) \\ \mathrm{C} 8-\mathrm{O} 1-\mathrm{C} 4-\mathrm{C} 5 & 89.1(4) \\ \mathrm{C} 3-\mathrm{C} 4-\mathrm{C} 5-\mathrm{C} 6 & 0.1(6)\end{array}$

$\mathrm{Br} 1-\mathrm{C} 1-\mathrm{C} 6-\mathrm{C} 5$
$\mathrm{C} 4-\mathrm{C} 5-\mathrm{C} 6-\mathrm{C} 1$
$\mathrm{C} 9-\mathrm{C} 5-\mathrm{C} 6-\mathrm{C} 1$
$\mathrm{C} 4-\mathrm{C} 3-\mathrm{C} 7-\mathrm{Br} 2$
$\mathrm{C} 2-\mathrm{C} 3-\mathrm{C} 7-\mathrm{Br} 2$
$\mathrm{C} 6-\mathrm{C} 5-\mathrm{C} 9-\mathrm{Br} 3$
$\mathrm{C} 4-\mathrm{C} 5-\mathrm{C} 9-\mathrm{Br} 3$

$178.0(3)$

$1.0(6)$

$-179.6(4)$

$-105.0(4)$

$76.3(5)$

74.9 (4)

$-105.8(4)$

Hydrogen-bond geometry $\left(A,{ }^{\circ}\right)$ for (III)

\begin{tabular}{lllll}
\hline$D-\mathrm{H} \cdots A$ & $D-\mathrm{H}$ & $\mathrm{H} \cdots A$ & $D \cdots A$ & $D-\mathrm{H} \cdots A$ \\
\hline $\mathrm{C} 2-\mathrm{H} 2 \cdots \mathrm{Br}^{\mathrm{i}}$ & 0.95 & 2.99 & $3.863(5)$ & 154 \\
$\mathrm{C} 7-\mathrm{H} 7 A^{\cdots} \cdots \mathrm{Br}^{\mathrm{ii}}$ & 0.99 & 2.94 & $3.866(5)$ & 155 \\
\hline
\end{tabular}

Symmetry codes: (i) $x,-y+1, z-1 / 2$; (ii) $x, y+1, z$. 